\title{
Telomerase-associated protein TEP1 is differentially expressed in brain metastatic breast cancer.
}

Shahan Mamoor

East Islip, NY USA ${ }^{1}$

1shahanmamoor@gmail.com

Metastasis to the brain is a clinical problem in patients with breast cancer ${ }^{1-3}$. We mined published microarray data 4,5 to compare primary and metastatic tumor transcriptomes to discover genes associated with brain metastasis in patients with metastatic breast cancer. We found that the telomerase-associated protein encoded by TEP $1{ }^{6}$ was among the genes whose expression was most different in the brain metastases of patients with brain metastatic breast cancer as compared to primary tumors of the breast. TEP1 may be relevant to processes underlying metastasis of primary tumor-derived cancer cells to the brain in humans with metastatic breast cancer.

Keywords: breast cancer, brain metastases, central nervous system metastases, TEP1, telomeraseassociated protein, systems biology of breast cancer, targeted therapeutics in breast cancer. 
One report described a 34\% incidence of central nervous system metastases in trastuzumabtreated patients with breast cancer ${ }^{2}$. This alarmingly high frequency of CNS metastasis events demands an enhanced understanding of the transcriptional makeup of brain metastatic tissues to support identification of therapeutic targets, whether they are treatment related or not. We performed a global comparative analysis of primary and metastatic tumors in patients with brain metastatic breast cancer ${ }^{4,5}$. We discovered significant differential and decreased expression of TEP1 in brain metastatic tissues of patients with metastatic breast cancer.

\section{Methods}

We used datasets GSE1259894 and GSE526045 for this global differential gene expression analysis of brain metastatic breast cancer in conjunction with GEO2R. GSE125989 was generated using Affymetrix Human Genome U133A 2.0 Array technology with $n=16$ primary tumors from patients with breast cancer and $n=16$ brain metastases from patients with brain metastatic breast cancer. GSE52604 was generated using Whole Human Genome Microarray 4x44K G4112F technology with $n=10$ normal breast tissues and $n=35$ brain metastases from patients with breast cancer, analysis performed using platform GPL6480. GSE43837 was generated using Affymetrix Human X3P Array technology with $n=19$ primary breast tumors and $n=19$ brain metastases from patients with breast cancer, analysis performed using platform GPL1352. The Benjamini and Hochberg method of $p$-value adjustment was used for ranking of differential expression but raw $p$-values were used to assess statistical significance of global differential expression. Log-transformation of data was auto-detected, and the NCBI generated category of platform annotation was used. A statistical test was performed to evaluate whether TEP1 gene expression was significantly between primary breast tumors and brain metastases in humans with breast cancer using a two-tailed, unpaired t-test with Welch's correction. We used PRISM for all statistical analyses of differential gene expression in human breast cancer (Version 8.4.0)(455). For Kaplan-Meier survival analysis, we used the Kaplan-Meier plotter online tool ${ }^{7}$ for correlation of TEP1 mRNA expression levels with relapse (recurrence)-free survival (RFS) in $n=3951$ breast cancer patients.

\section{Results}

We performed global comparative transcriptome analysis of the primary and metastatic tumor tissues of patients with brain metastatic breast cancer using published microarray data ${ }^{4-6}$ to describe the transcriptional landscape of brain metastasis in human breast cancer in an unbiased fashion and for the discovery of novel therapeutic targets.

\section{TEP1 is differentially expressed in the brain metastases of patients with brain metastatic breast} cancer.

We identified gene encoding the telomerase-associated protein, TEP1, as among the genes whose expression was most significantly different in the brain metastases of patients with brain metastatic breast cancer $^{4}$ (Table 1). When sorting each of the genes expressed in brain metastases based on significance of difference as compared to the primary tumors of patients with brain metastatic breast cancer, TEP1 ranked 236 out of 22297 total transcripts (Table 1), equating to $99.0 \%$ differential expression. Differential expression of TEP1 in the brain metastases of patients with brain metastatic breast cancer was statistically significant (Table $1 ; p=2 \mathrm{E}-03$ ).

Cross-validation of differentially expressed genes across datasets in cancer can be difficult and more challenging than when cross-validating across organs and sorted cell populations due to higher 
levels of heterogeneity in tumors, and differing methods of tumor sampling between laboratories. Thus, we asked whether we could validate differential expression of TEP1 when comparing brain metastases

from patients with breast cancer to normal breast tissue as opposed to primary tumors of the breast. Comparison of global gene expression profiles of 10 normal breast tissues to 35 brain metastases 5 revealed that TEP1 was among the genes whose expression was most significantly different transcriptome-wide between the breast and brain metastases in patients with breast cancer (Table 2). When sorting each of the genes expressed in brain metastases based on significance of difference in expression between brain metastases and normal breast tissues, TEP1 ranked 8251 out of 41093 total transcripts (Table 2), equating to $80.0 \%$ differential expression. Differential expression of TEP1 in brain metastases from patients with brain metastatic breast cancer in this dataset was statistically significant (Table $2 ; p=2.09 \mathrm{E}-03$ ).

\section{TEP1 is expressed at significantly lower levels in the brain metastases of patients with brain metastatic breast cancer.}

PAGE 3 brain metastasis of patients with brain metastatic breast cancer to determine magnitude and direction of change in TEP1 expression in brain metastatic tissues. TEP1 was expressed at lower levels in the brain metastases of patients with breast cancer as compared to primary tumors of the breast, and this difference was statistically significant (Figure $1 ; p=0.0014$ ). We calculated a mean fold change of $0.73 \pm 0.25$ in TEP1 expression when comparing primary tumors of the breast to brain metastatic tissues in patients with breast cancer (Table 1).

\section{TEP1 expression associates with survival outcomes in breast cancer.}

We performed Kaplan-Meier survival analysis to evaluate correlation between TEP1 mRNA expression levels with survival outcomes in patients with breast cancer. In 3951 breast cancer patients, we found a statistically significant correlation between TEP1 expression and relapse (recurrence)-free survival (RFS) (Figure 2; log rank $p$-value: $<1 \mathrm{e}-16$ for relapse-free survival, hazard ratio: 0.48 (0.41 0.56) (Fig. 2)). Lower TEP1 mRNA levels in primary tumors of the breast, like which we observed in metastases to the brain, were a negative prognostic indicator in patients with breast cancer. Median RFS was 25 months for patients with low tumor expression of TEP1 while median RFS was 61 months for patients with high tumor expression of TEP1 (Table 3).

We also performed Kaplan-Meier survival analysis to evaluate correlation between TEP1 mRNA expression levels with overall survival outcomes in patients with breast cancer. In 1402 breast cancer patients, we found a statistically significant correlation between TEP1 expression and overall survival (OS) (Figure 3; log rank $p$-value: 0.0027 for overall survival, hazard ratio: 0.62 (0.45 - 0.85) (Fig. 3)). Lower TEP1 mRNA levels in primary tumors of the breast, like which we observed in metastases to the brain, were a negative prognostic indicator in patients with breast cancer. Median OS was 62.07 months for patients with low tumor expression of TEP1 while median OS was 110.4 months for patients with high tumor expression of TEP1 (Table 4).

Thus, by mining published microarray data ${ }^{4-6}$ in an unbiased and systematic fashion, we identified TEP1 as among the genes whose expression was most different in the brain metastases of patients with breast cancer both when compared to primary tumors of the breast and to the normal breast; TEP1 was expressed at significantly lower levels in brain metastases as compared to primary tumors of the breast. TEP1 expression in primary tumors of the breast in a large cohort of patients demonstrated a significant correlation with relapse-free and overall survival, with higher expression of TEP1 correlating with improved survival in humans with breast cancer. 


\section{Discussion}

TEP1, also known as TP1, was originally identified by cloning of a homolog of mammalian telomerase, and shares similarity to the telomerase protein from Tetrahymena p806. TEP1 likely physically associates with telomerase as immunoprecipitation of TEP1 could also pulldown telomerase ${ }^{6}$. One study of TEP1-deficient mice suggested that TEP1 was not required for the activity of telomerase nor for the maintenance of its length ${ }^{8}$. TEP 1 can interact with vRNA, a component of the vault particle, ribonucleoprotein complexes with poorly described function but suggested to participate in assembly of the nuclear pore complex ${ }^{8-10}$. TEP1 interacts with multiple human vault RNAs, which may occur through WD40 domains in the carboxy-terminus of TEP1 ${ }^{10}$. In TEP1-deficient mice, the cap of the vault is less dense, and deficiency of TEP1 abrogated association between vault particle and vault RNA ${ }^{11}$. Thus, TEP1 interacts with telomerase, may not be required for function of telomerase, associates with multiple vault RNAs of the vault particle and stabilizes association between vault RNAs and the vault particle, a molecule with poorly described function but suggested to participate in assembly of the nuclear pore complex.

We report here that TEP1 is among the genes whose expression is most different in the brain metastases of patients with brain metastatic breast cancer and that its expression in primary tumors of the breast correlated with patient survival. Understanding the role of TEP1 in cell biology of the breast and in the brain in greater detail may provide insights into molecular mechanisms underlying metastasis to the brain in patients with metastatic breast cancer. 


\section{References}

1. Lin, N.U., Amiri-Kordestani, L., Palmieri, D., Liewehr, D.J. and Steeg, P.S., 2013. CNS metastases in breast cancer: old challenge, new frontiers.

2. Bendell, J.C., Domchek, S.M., Burstein, H.J., Harris, L., Younger, J., Kuter, I., Bunnell, C., Rue, M., Gelman, R. and Winer, E., 2003. Central nervous system metastases in women who receive trastuzumab-based therapy for metastatic breast carcinoma. Cancer, 97(12), pp.2972-2977.

3. Tsukada, Y., Fouad, A., Pickren, J.W. and Lane, W.W., 1983. Central nervous system metastasis from breast carcinoma autopsy study. Cancer, 52(12), pp.2349-2354.

4. Iwamoto, T., Niikura, N., Ogiya, R., Yasojima, H., Watanabe, K.I., Kanbayashi, C., Tsuneizumi, M., Matsui, A., Fujisawa, T., Iwasa, T. and Shien, T., 2019. Distinct gene expression profiles between primary breast cancers and brain metastases from pair-matched samples. Scientific reports, $9(1)$, pp. $1-8$.

5. Salhia, B., Kiefer, J., Ross, J.T., Metapally, R., Martinez, R.A., Johnson, K.N., DiPerna, D.M., Paquette, K.M., Jung, S., Nasser, S. and Wallstrom, G., 2014. Integrated genomic and epigenomic analysis of breast cancer brain metastasis. PloS one, $9(1)$, p.e85448.

6. Harrington, L., McPhail, T., Mar, V., Zhou, W., Oulton, R., Bass, M.B., Arruda, I. and Robinson, M.O., 1997. A mammalian telomerase-associated protein. Science, 275(5302), pp.973-977.

7. Györffy, B., Lanczky, A., Eklund, A.C., Denkert, C., Budczies, J., Li, Q. and Szallasi, Z., 2010. An online survival analysis tool to rapidly assess the effect of 22,277 genes on breast cancer prognosis using microarray data of 1,809 patients. Breast cancer research and treatment, 123(3), pp.725-731.

8. Liu, Y., Snow, B.E., Hande, M.P., Baerlocher, G., Kickhoefer, V.A., Yeung, D., Wakeham, A., Itie, A., Siderovski, D.P., Lansdorp, P.M. and Robinson, M.O., 2000. Telomerase-associated protein TEP1 is not essential for telomerase activity or telomere length maintenance in vivo. Molecular and cellular biology, 20(21), pp.8178-8184.

9. Kickhoefer, V.A., Stephen, A.G., Harrington, L., Robinson, M.O. and Rome, L.H., 1999. Vaults and telomerase share a common subunit, TEP1. Journal of Biological Chemistry, 274(46), pp. $32712-32717$.

10.Vollmar, F., Hacker, C., Zahedi, R.P., Sickmann, A., Ewald, A., Scheer, U. and Dabauvalle, M.C., 2009. Assembly of nuclear pore complexes mediated by major vault protein. Journal of cell science, 122(6), pp.780-786.

11.Kickhoefer, V.A., Liu, Y., Kong, L.B., Snow, B.E., Stewart, P.L., Harrington, L. and Rome, L.H., 2001. The Telomerase/vault-associated protein TEP1 is required for vault RNA stability and its association with the vault particle. The Journal of cell biology, 152(1), pp.157-164. 
Figure 1: TEP1 is expressed at significantly lower levels in the brain metastases of patients with brain metastatic breast cancer.

The mRNA expression level of TEP1 in primary tumors of the breast (left) and in the brain metastases of brain metastatic breast cancer patients (right) is graphically represented here with mean TEP1 levels marked and the result of a statistical test evaluating difference in mRNA expression level between brain metastases and primary tumors of the breast, a $p$-value, listed above. 
Figure 2: TEP1 expression correlates with relapse-free survival in patients with breast cancer.

Depicted in this Kaplan-Meier plot is the probability of relapse (recurrence)-free survival (RFS) for $n=3951$ total patients stratified into two groups, based on low or high expression of TEP1 in patient primary tumors. The log rank $p$-value denoting statistical significance of difference in relapse-free survival when comparing the two groups, as well as hazard ratio for this comparison is listed above. Listed below is the number of patients at risk (number of patients alive) per interval, after stratification based on TEP1 expression; in the first interval, number at risk is number of patients alive; in each subsequent interval, number at risk is the number at risk less those who have expired or are censored. 


\section{Low expression cohort (months)}

25

High expression cohort (months)

Table 3: Median relapse-free survival is superior in breast cancer patients with high primary tumor expression of TEP1.

The relapse-free survival of $n=3951$ breast cancer patients based on stratification into low or high tumor expression of TEP1 is listed in this chart. 
TEP1 (228670_at)

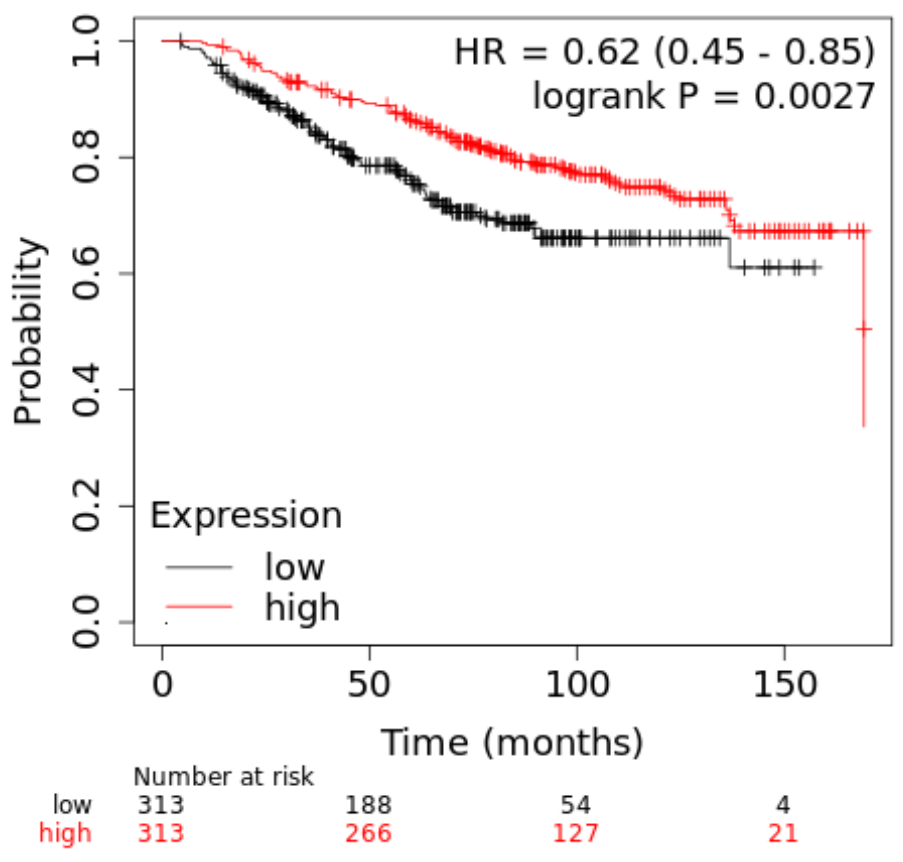

Figure 3: TEP1 expression correlates with overall survival in patients with breast cancer.

Depicted in this Kaplan-Meier plot is the probability of overall survival (OS) for $n=1402$ total patients stratified into two groups, based on low or high expression of TEP1 in patient primary tumors. The log rank $p$-value denoting statistical significance of difference in overall survival when comparing the two groups, as well as hazard ratio for this comparison is listed above. Listed below is the number of patients at risk (number of patients alive) per interval, after stratification based on TEP1 expression; in the first interval, number at risk is number of patients alive; in each subsequent interval, number at risk is the number at risk less those who have expired or are censored.

PAGE 11 
Table 4: Median overall survival is superior in breast cancer patients with high primary tumor expression of TEP1.

The overall survival of $n=1402$ breast cancer patients based on stratification into low or high tumor expression of TEP1 is listed in this chart.

PAGE 12 